\author{
DEPARTAMFN'TO DF, INDOSTIRIA, INSPEÇÕO E CONSERVAÇAO DOS \\ PRODU'IOS ALIMENTICIOS DE ORIGEM ANIMAI. \\ Diretur: P'rof. Dr. P'. Mucciolo
}

\title{
UN APPAREIL POUR LA MESURE EN CONTINU DE L'ABSORPTION D'OXYGÈNE (")
}

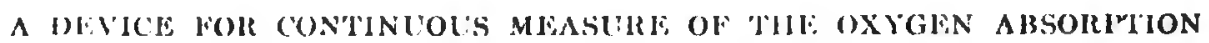

\section{LUCIEN Kr:HREN}

Docteur es Scienees de l'Lmiversili de Puris

Bulsista do ('ousclho Nacional de P'eseyuisas

3 planches 13 lixures)

\section{INTROIDUCIION}

la mesurc de l'absorption d'oxygene est d"une grande importance en biologic a co hiochimie animale el vegrétable. Iille a servi de base aux études sur la respiration des lissus, des microorganismes, el sur l'autoxydation des lipides. Les irçlıodes utilisées jusqu'à présent peuvent se diviser en deux groupes:

I) Méthodes eudiométrigues: - Ou soutire l'air qui était en contact avec le tiśn on la plante dans un récipient fermé. Cel air est alialysé dans un cudiomile, oi se delerminent, en premier lieu, un volume exact flair, puis la quantile de gaz cardonique de celui-ci, par passage sur de la potasse, et mesure

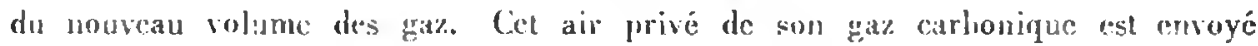
sur du plosiphore, ou sur du pyrogallol, qui relient loxygìne. Lat notveau velunse oceupé par les gaz cst mesuic pour connatre, par différence, la guantilé doxygerne qui étail présente daus ect air.

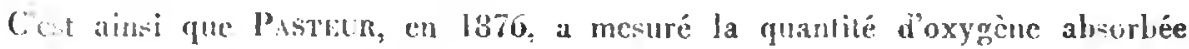
par la levur: en aćrobic. C"est surtoul en hiolugic végitale, pour mesurer la respiration de végétaux, que la méthode cudiométrique a été la plus sonvent cmployéc. lijen que précise, celte méthode, par excmple appliquée avec licxcollent cudiomitre de Piscrtilon, se prête difficilement a l'étude de trop petiles guantités de matière. lille ne permet naturellement pas de suivre la cinélique ale la ríaction de l'ahsorption de l'oxygrinc. De plus la concentration des

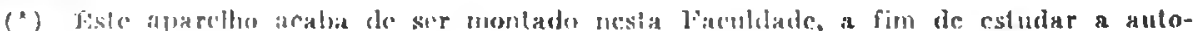

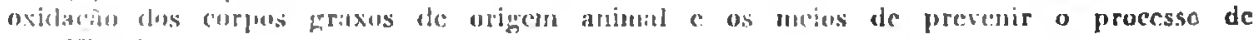
ranciôicuşitu. 
gaz dans l'air, qui est au contact avec les tissus, varie an cours de l'expéricuce. I.a míthode eudiométrique n’est pratiquement pas utilisé pour la mesure de l'al. sorption d'oxygene par les lipides.

1I) Méthodes manométriques: - Manquant de précision dans les cas du. macro-mesures, ces méthodes sont moins utilisées, sauf en ce qui concernc la micro-méthode de Warburc. Celle ei est d'une grande précision et d'une haute sensibilité. elle est largement utilisée en biologie. Fille a servi à son auteur pour réaliser ses importants travaux, notamment ses éludes du métabolismi: des lissus cancércux. L'appareil de RARBLRG est un micro manometre en L, garmi dinn lieguide spériai tenant en dissolution un sel biliaire pour faciliter le mouil. lage des parois. I.a branche droite du manomitre est en rélation avec le récipient où se trouve le produit à étudier, ainsi qu'une pastille de potanse placé dans une alvéole. Le's deux branches du manomitre ahoutissent en lias it un réservir de caoutchouc muni d'une vis de pression réglable. Le récipient oi s'effectue l'absorption doxygine est immergé dans une curve thermostatique it la température stabiliséc au $1 / 10^{\prime \prime}$ de degré $(.$.

Principe: le volume gazeux élant maintenu conslant, les gaz disparus fon formés) modifient la pression. Pour la lecture, on ramene le nivean du liquide dans la branche de droit a une hauteur jefentique a chaque nesure, en agrisant sur la vis du réservoir, et on note la hauteur atleinte par la colome de: linquide dans lat branche de gauche. Selon lintensite de la dipression on bivcan desceud.

Fin ce qui concerno lélude de l'autoxydation des lipides: cel appareil a che utilisé par quelques anteurs. Salistaisant dans la rerherehe sciembilique, l'ap-

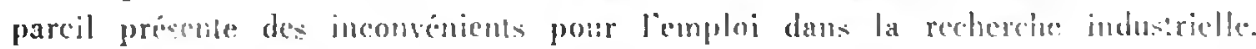

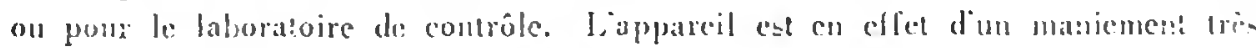
délicat, sa sensibilite est trop pousse pour la mesure de colle sorte de réadion qu'cst labsorption doxygine par les corps gras. Il ne lonctionne qu'ares de tris perites quantilis de matione seulemeit.

An slade de: la macro-methode, er pour l'etude de lautoxydation, on pent

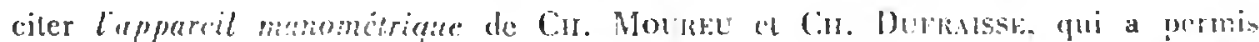
à ses auteurs leur grande dénonstration de lat généralite du phénomene antioxy. gène. Cess un appareil à mesures comparatives. Il se compose de tubes ha. rométriques aboulissant à la base à um reservoir de mereme. A chaque cxtrí mile des lubes, on soude une amponle recourbée qui contient le produit que l'on veut indier. Aulant de produits, autant de tubes. Liair est remplace dans les tulas par de loxyginare.

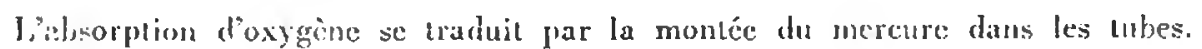
On peut reprocher à cet appareil de ne donner pratiquencent que des indications 
comparalives et relatives, d'exiger pour son fonctionnement d'assez grandes quanlités de matière, el de ne travailler qu'en atmosphère d'oxygène.

les modalités ress appareils que nuus benons de voir. du point de vue fondionmentent sont:

1) Findiometre: Tahsorption dioxgenente pendan la réaction ne peutêtre suivie cinétiquement. lappareil ne donnant que le résultat final. L'absorption doxygane se fait dans une almosphere dont la pression varie. Macrométhode.

2) Warburg: lahorpution doxygene peuteples suivie au cours du temps, elle or ralise daus une atmosphere i iolume constant. mais à pression variable. Sirulesnent mirro-molhode.

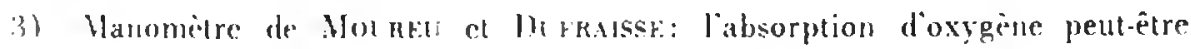
suivic au cours du temps, mais sentement à titre romparatif. Flle a lieu à volume variable, "l a pression relativement constant. Macro-méthode.

lin considerant ces lypes dapparibs, il paraissail infiressunt de mettre au point un apparcil qui promettre de mesurer au cours du temps sans les incomvénients ciles plus haut. labsorption doxygene. lin conséquence cet appareil levail:

- permetire de suivre la cinélique de la réaction;

- realiser celle réaction à pression el à volume conslants;

- être de maniement simple, fonctionner automatiquement;

aborder la macro-méthude. et la semi micro.méthode, suivint la guantile de matière à meltre con elude.

I'n appareil de ce lype a été imagine par Swiviter pour l'étude de l'oxy. dation du caoutchouc, el appliqué à las mesure de lautoxydation des esters de Thuile de palme par loom et llet.lur. Cel appareil présente quelques défants: sa forme peu pratique. te dispositif du manomètre à contacls. qui ne permet pas de controler el de regler la sensibilisi de lappareil.

Lappareil que je présente ici est done basé sur le principe de l'appareil do Survort. mais comporte des motifications telles que les incouvénients du modele primitif ont éle élimines.

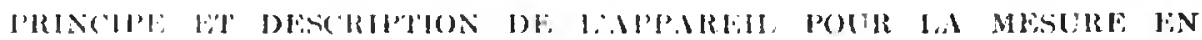

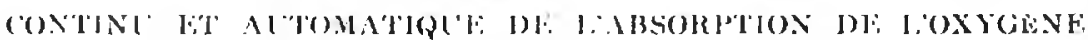

Principe: la quantiti doxygène absorbée esl automaliquement compensée par la produclion diune quantité équivalente à l'aide d'une cellule à électrolyse, landis quine cellule jdenlique enregistre volumétriquement cette production. 
Electrolyse: la production d'oxygène est assurée par l'électrolyse d'une solution saturée de sulfate de cuivre, entre un cathode de mercure et une anode de platine, sous une tension de 6 ou 12 volts. Le cuivre se dissout dans le mercure al fur et à mesure de son apparition, en donnant, un amalgame, et il se dégage de l'oxygène pur à l'anode, suivant la réaction (simplifiée):

$$
\begin{aligned}
& \mathrm{SO}_{4} \mathrm{CH} \longrightarrow \mathrm{SO}_{4}+\mathrm{Cu} \\
& \mathrm{SO}_{4}+\mathrm{H}_{2} \mathrm{O} \longrightarrow \mathrm{SO}_{4} \mathrm{H}_{2}+\mathrm{O}^{+}
\end{aligned}
$$

SAlvetTr avait vérifié qu'il ne se produisait aucun dégagement d'hydrogène à la cathode tant que la solution de sulfate de cuivre restait suffisamment concentrée, c'est-à-dire tant que l'électrolyse secondaire de l'acide sulfurique, produit par la première électrolyse, n’avait pas lieu. La décoloration de la solution de sulfate de cuivre permet de prévoir l'épuisement de celle ci. J'ai aussi vérifié que l'oxygène produit était exempt d'ozone lors de lílectrolysc normale.

Apparcillage: - Il se compose de:

1) Une cuve à eau dont la températurc est maintenue ronstante par un thermostat à un $1 / 5^{\circ}$ de degré C. près. Tous les appareils baignent dans cette cuve. La température peut-être comprise entre 20 et 50 degrés $C$, suivant less conditions de l'expérience.

2) Un couple, ou des couples de cellules à électrolyse. Le, couple comprend:

a) Lne cellule dite compensutrice (figure 2). ("cst un récipient de verre Pyrex de $2,5 \times 8 \mathrm{~cm}$, pourvu d'un capuchon rodé par lequel passent l'électrode de platine et lélectrode de tungstène. La première plonge dans la solution de sulfate de cuivre, l'autre dans le mercure. Le haut de l'appareil communique avec le récipient destiné à recevoir las sulstance que l'on veut étudier. Ine alvéole est prévue sur le côté de ce récipient pour y recevoir un peu de polasse (pour absorber le $\mathrm{CO}_{2}$ éventucllement formé). Un conduit à robinet rodé est prévu pour pouvoir évacuer l'air contenu dans le récipient afin de le cemplacer pas do l'oxygène dans le cas de certaines expériences. P'our cela il suffit de brancher l'apparcil sur un tube de trompe à cau muni latéralment d'un manomètre ordinaire. La vide étant fait (on n'oublie pas d'oblurer pendant cette opération la branche de gauche du manomitre à contacts), on branche lés électro. des dans le circuit de l'accumulateur; il se dégage de l'oxygène dans la cellule jusqu'au moment où le manomètre extérieur indique que la pression initiale a ćté rétablie par l'oxygène produit. On enlève alors le tube à vide, après avoir 
fermé le robinet rodé, et on dégage la branche du manomètre à contacts que l'on avait obturéc.

La cellule est terminée par le las par un tube recourbé qui forme vase communiquant avec elle, et qui aboutit a un tube en $U$ contenant du mercure. La hranche de gauche de ce tube est ferméc hermitiquement par un houchon traversé par une vis manouvrable, sur laquelle est soudéc un fil de contact. L'autre branche est ouverte à l'air libre, un fil de cuivre y pénétre et plonge dans lc mercure.

b) Uńe cellule dite enregistrcuse (figure 3). Elle est de mêmes dimensions que la première cellule, mais elle ne comporte ni manometre à contacts, ni récipient il expériences. Le haut de celte cellule communique seulement avec une burette graduce en $1 / 10^{\prime \prime}$ de cc, plongeant dans la cuve à euu.

3) Lin accumulateur de 6 volls ou de 12 volts: - Fonctionnement: quand la malière en expérience absorbe de l'oxygène, une légère dépression se produit dans la collule qui a pour conséquence de déséquilibrer le niveau du mercure dans lis branches du manometre à contacts. La colonne de mercure B (figure 1) remonte et contacte le fil A. Ainsi le circuit électrique des deux cellules est ferme, la cellule compensatrice débite la quantité d'oxygène nécessaire pour rétablir la pression initiale qui ixjstait dans le récipient avant l'absorption d'oxygrine. Dis que cette pression est à nouveau atleinte, la colonne de mercure $B$ retonthe à sa place de départ, et de par cela interrompt le contact avec le fil A. La quantité d'oxygìne absorbce par la substance en expérience a donc illi remplacée par une quantité égale. Comme la cellule enregistreuse fonctine en même temps, et identiquement, que la cellule compensatrice, il suffit de lire directement dans la bureite à eau de la cellule enregistreuse les vo. lumes doxygenc produits, lesquels correspondent aux volumes d'oxygène ab. sorbéa.

(1: peut régler la sensibilité du manomètre à contacts, en manœuvrant la vis C. (tigure 1) ce qui a pour effet de raccourcir ou d'éloigner l'espace à parcourir par la colonne de mercure $B$ pour fermer et interrompre le circuit électrique de l’apparcil.

Le riglage de cette vis permel de faire travailler l'appareil en macro ou ('n semi-micro mesures, suivant le type ou la quantité de la sulsslance a mettre cu expérience. On peut ainsi obtenir que les intermittences de contact entre A a $B$ sorrespondent à l'échelle du centième de cc oul du dixième de ce d'oxygène fabriqué et enregistré. Un graud avantage découlant de l'emploi de ce manoniètre réglable, c'est qu'il devient possible sans inconvénient d'arrêter momentanément la marche de l'appareil; pour cela, on remonte largement le fil A grace a la vis $C$, on ouvre le robincl du récipient communiquant avec la cel. 
lule compensatrice, dans lequel il est alors possible d'intraduire une substance nouvelle. On procide ensuite aux mêmes manipulations que lors de la mise en marche initiale de l'appareil. L'equilibre de température une fois alteinl, on rantene le fil $A$ à la position où il figurail précédemment. Ainsi larrêt de l'appareil n'a pas d'influence sur l'ensemble de la mesure. les quantiles doxygéne qui seront absorbées s'ajouteront simplement a la suite des précédents dans la cellule cnregistreuse.

\section{FXYMPIE DITUASTION}

Mesure de labsorplion d'oxygene par un corps gras: - la mesure au cours du lemps de l'auloxydation des corps gras. par détermination des quantités doxygene quils absorbent, est primordiale dans ce genre diblude. I,e pré. sent appareil ş prête parfaitement bien. Il suffit de placrer une quantité de corps gras. par exemple l gramme, de préférence étendu sur un rouleau de papier filtre pour offrir une plus grande surface au contact de lair. on de l'oxygène, daus le réciugient prévu à cet fffet. Après aroir clos soigneusemen! les appareils; introduit leau dans la burette de la cellule conregislreuse justuau zéro, attendu que la température se soit égalisée dans les apparcils, ou ancine le fil $A$ a une distance de 1 . mm environ du niveau de la colonne de mercure $\mathrm{B}$. Il sullit de lire aux intervalles désiré:, le volume doxygence que le corps gras absorthe, dans la burette de la cellule enregistreusc. Il cst facile diuscrive la courbe de celle absorption en fonction du temps.

Autres ulilisations: - l'appareil peut servir à la mesure des respiralions: levure, pelites plantes, morceaux de lissus, etc. Il peut aussi sersir a meciper la respiration de petits animaux comme des insectes, à étudier la résislance à l'oxydation des mélaux, du caoutchouc, des huiles.

\section{(ONCIASIONS}

Jai présenté un apparcil de mesure de l'absorplion doxygene possérlant les caractéristiques suivantes:

a) fonctionne en continu et automatiquement

h) la réaction se fait à volume el à pression constants

c) le volume d'oxygine absorbé se lit directement, sans calculs

d) les expériences peuvent être conduites à l"échelle de la macro ou de la semi-!nicro méthode 
e) il est possible d'interwmpre sans dammage pour l'ctude de la cinétique de la réaction le Conctionnement de l'appareil. et pour suivrc les mestures.

Les applicalions de cet appareil peuvent être nombreuses: mesures do la respiration des levures, des lissus, de petits animaux, de lauloxydation des corps gras, de loxydalion des mélaux, ctc.. Son automalisme, la simplicité de son fonctionncment, la lecture directe des résuliats, son adaplation à limportance re l'ćchantillon que l'on veut étudier, le désignent particulièrentul pour le laboratoire de contrôle el d'analyse, el pour les essais inristriels.

Je liens à remercier le Professeur Libcralli qui ma offert l'hospilalice de son laboratoire de Pharmacic Caléniçü, de la laculté de Pharmacie et d'Odiontologric de f'Cniversité de S. Paulo, pour $y$ effectucr les premiers essais de lappareil.

BIHISIOGRAPHIL:

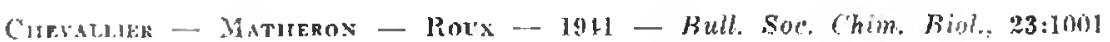

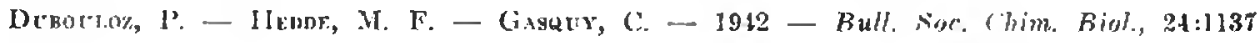

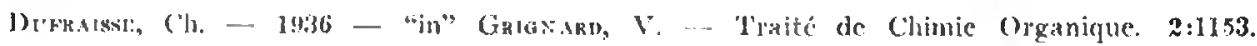
Paris

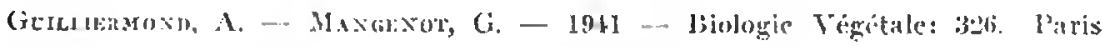

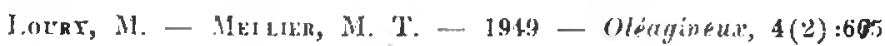

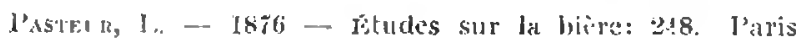




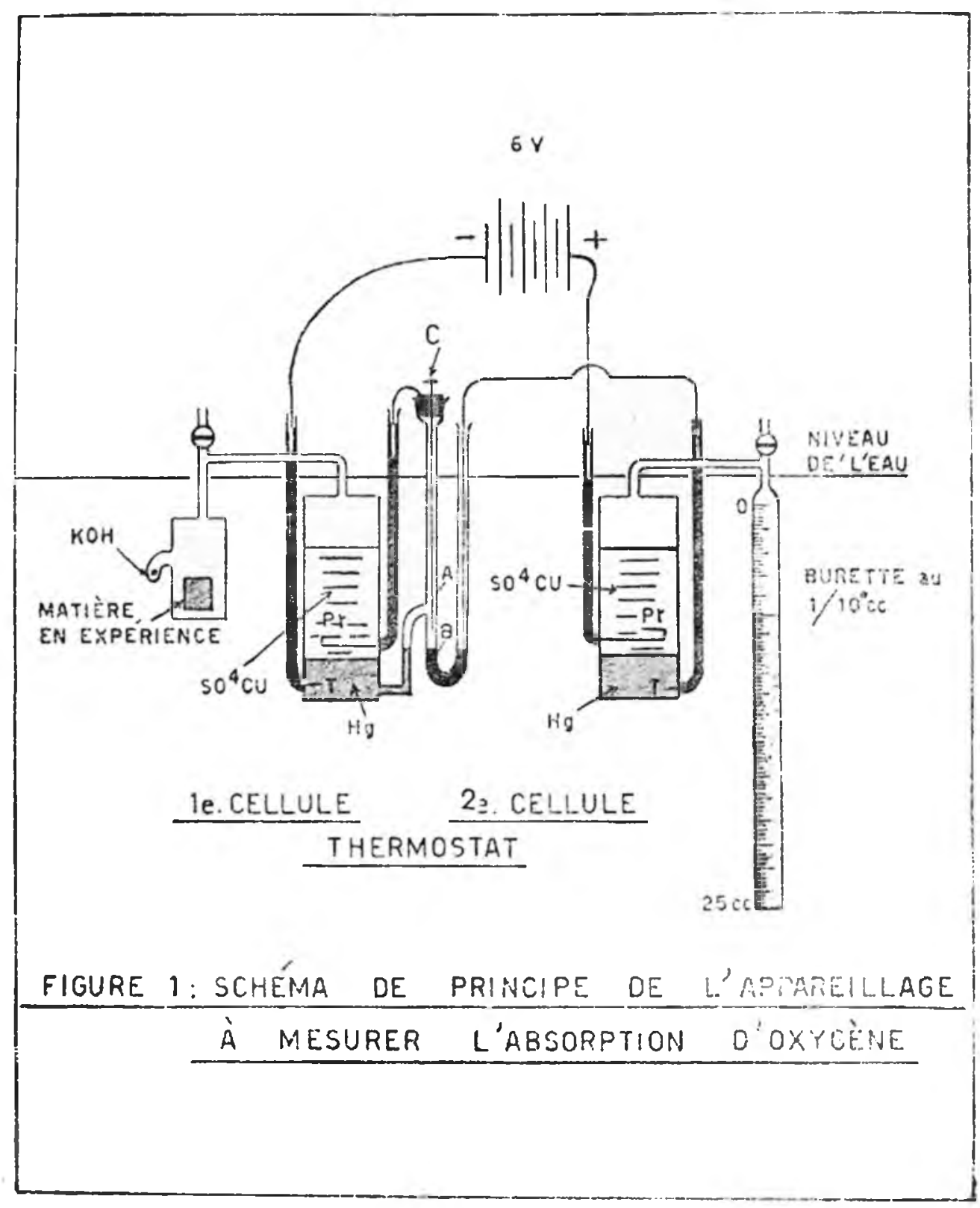




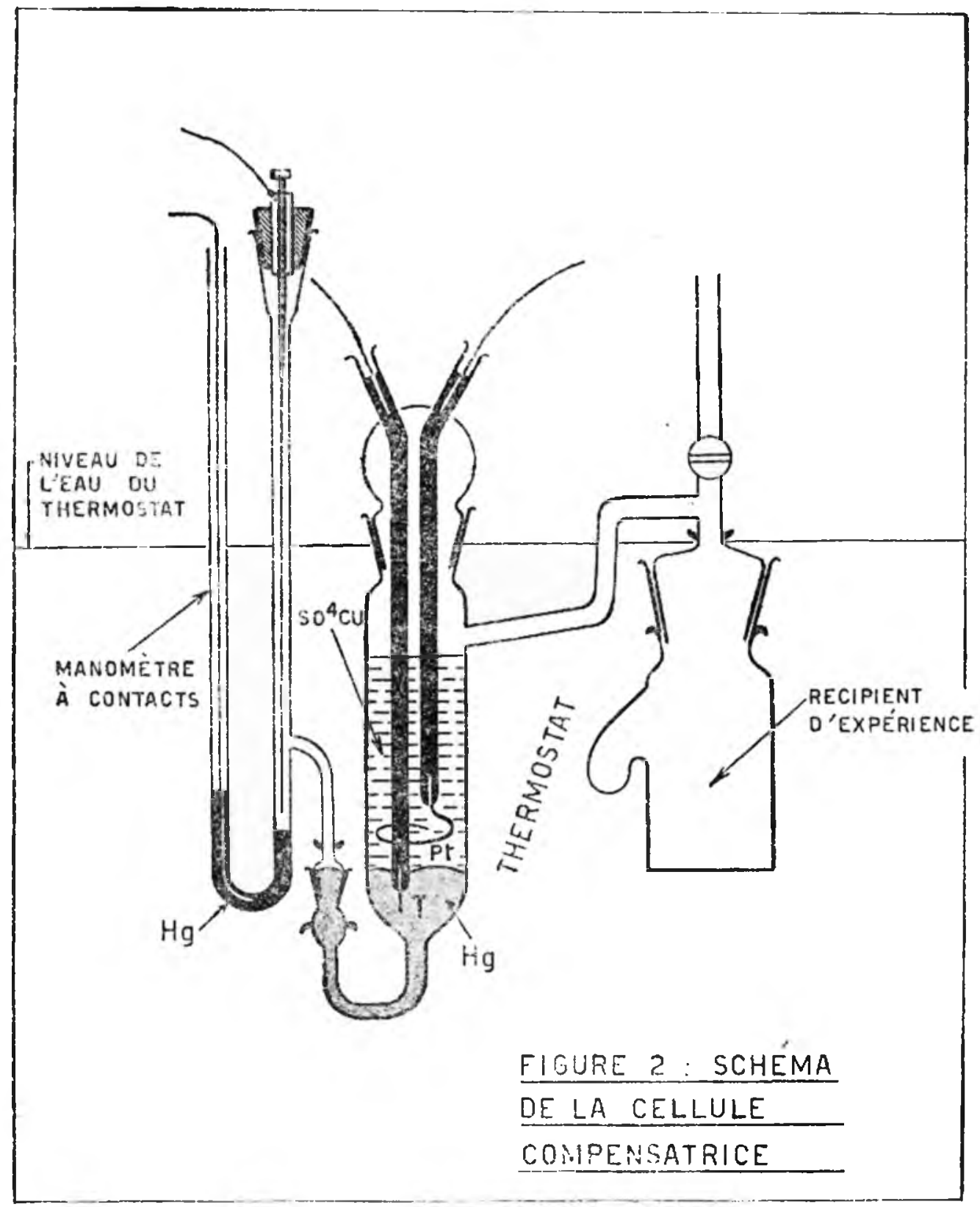




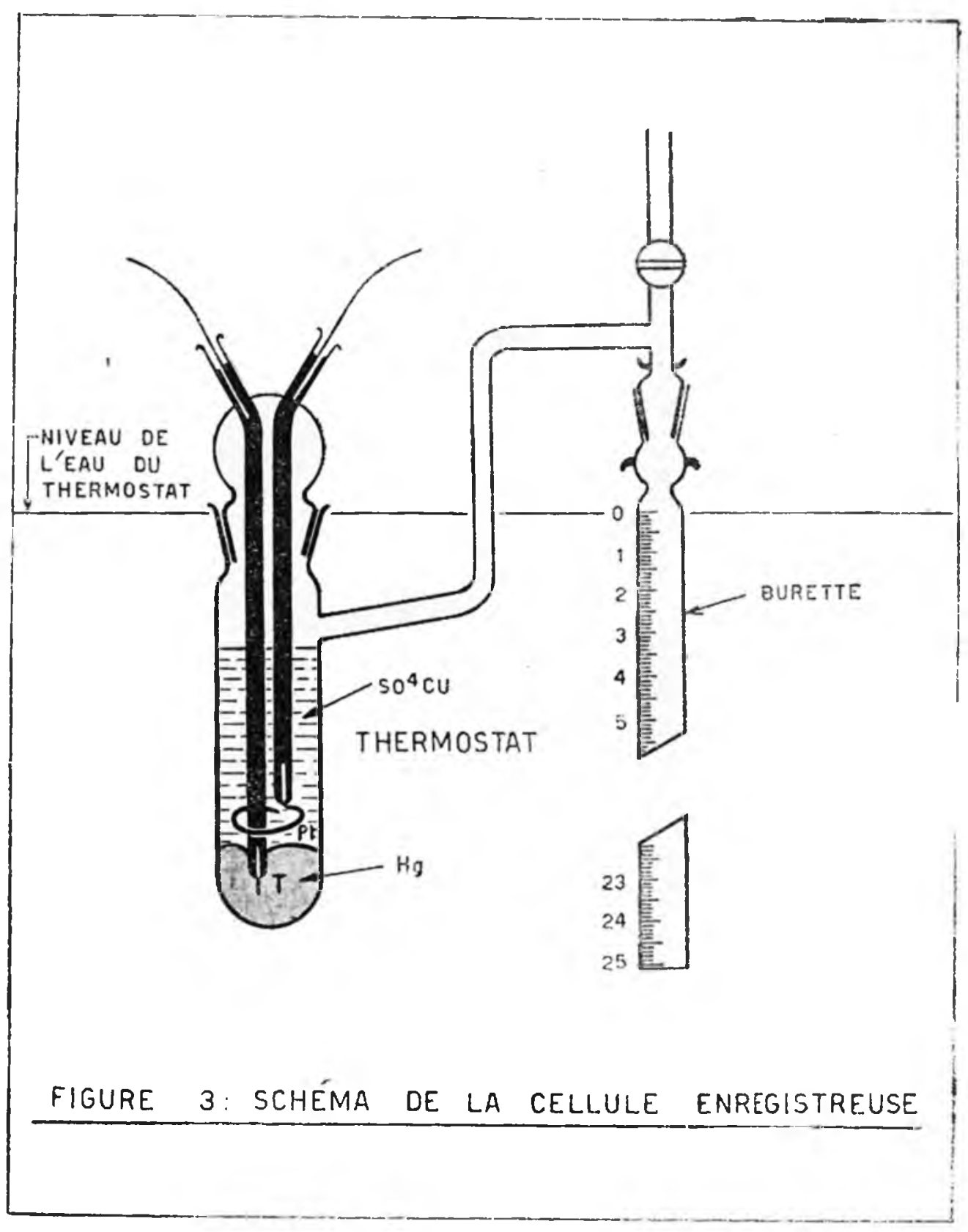

